\title{
Pancreatic ductal adenocarcinoma with inferior vena cava invasion: a report of three resected cases
}

\author{
Takuya Mizumoto, Tadahiro Goto, Hirochika Toyama*, Keitaro Sofue, Sadaki Asari, Sachio Terai, Motofumi Tanaka, \\ Masahiro Kido, Tetsuo Ajiki, Takumi Fukumoto and Yonson Ku
}

\begin{abstract}
Background: Pancreatic ductal adenocarcinoma (PDAC) often infiltrates to the adjacent major vasculatures; however, direct invasion of PDAC to the inferior vena cava (IVC) is uncommon.

Case presentation: We report our experience with three cases of PDAC directly invading the IVC wall. All three patients underwent pancreatoduodenectomy along with wedge resection of the IVC wall without severe postoperative complications. Histopathological studies revealed tumor infiltration to the adventitia of the IVC. All patients achieved negative surgical margins. One patient was still alive 26 months after surgery without tumor recurrence. Two patients experienced recurrence; one patient experienced liver metastasis but was still alive and in a stable condition without further tumor progression 12 months after surgery. Another patient experienced multiple liver metastasis 10 months after surgery and died 26 months after surgery.

Conclusions: Pancreatoduodenectomy along with wedge resection of the IVC wall for patients with PDAC directly invading the adventitia of the IVC can be performed safely. Further accumulation of cases is needed to elucidate the prognostic impact of IVC invasion.
\end{abstract}

Keywords: Pancreatic ductal adenocarcinoma, Inferior vena cava, Invasion, Resection

\section{Background}

Pancreatic ductal adenocarcinoma (PDAC) has the worst prognosis among all gastrointestinal cancers. Surgical resection is the only possibly curative therapy; however, only 15 to $20 \%$ of pancreatic cancer is indicated for surgery with curative intent [1,2]. This is due to not only distant metastasis but also local invasion to adjacent organs. PDAC frequently infiltrates the major vasculatures that exist posterior to the pancreas, such as the superior mesenteric artery, portal vein (PV), superior mesenteric vein (SMV), or common hepatic artery. These findings are considered significant in regulating the resectability of the tumors $[3,4]$. However, direct invasion of the inferior vena

\footnotetext{
*Correspondence: tymhr@me.com

Division of Hepato-Biliary-Pancreatic Surgery, Department of Surgery, Kobe University Graduate School of Medicine, 7-5-2, Kusunoki-cho, Chuo-ku, Kobe 650-0017, Hyogo, Japan
}

\section{Case presentation}

Case 1

A 50-year-old man presented at our hospital with upper abdominal pain and loss of appetite. Computed tomography (CT) imaging demonstrated a hypovascular mass that measured $10 \mathrm{~mm}$ in diameter in the uncinate process of the pancreas. The dense soft tissue of 


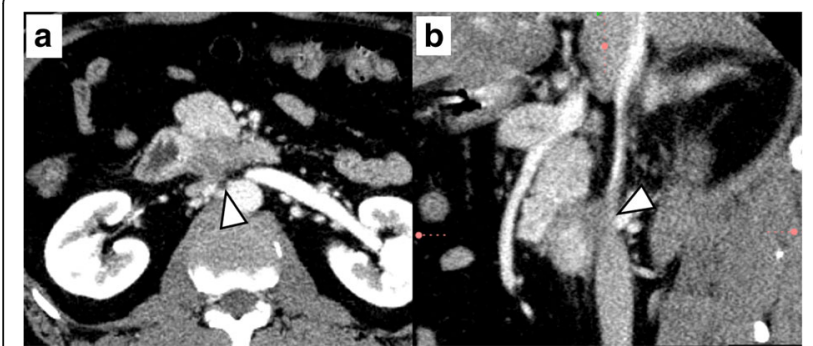

Fig. 1 Preoperative computed tomography image of case 1. a Dense soft tissue of the pancreatic tumor contacting the ventral surface of the inferior vena cava (IVC) (arrowhead). b Sagittal multi-planar reformation showed that the interface between the tumor and the IVC was not well defined

the pancreatic lesion was in contact with the ventral surface of the IVC; however, signs of obvious invasion were not detected (Fig. 1). A self-expanding metallic stent was placed in the duodenum because the horizontal portion of the duodenum was obstructed by the tumor. Therefore, pancreatoduodenectomy (PD) was performed. During surgery, stiff attachment between the tumor and the IVC was identified and wedge resection of the IVC wall was performed via side clamping of the IVC (Fig. 2). Pathological studies of the surgical specimen revealed direct invasion by the PDAC to the adventitia of the IVC (Fig. 3). He was discharged without significant postoperative complications, including IVC thrombosis or leg edema, on postoperative day (POD) 25. He underwent adjuvant chemotherapy (S-1; $120 \mathrm{mg} /$ day) for 4 months; however, the CT imaging performed 6 months after surgery identified liver metastasis that was treated with systemic chemotherapy (Gemcitabine, $1000 \mathrm{mg} / \mathrm{m}^{2}$;

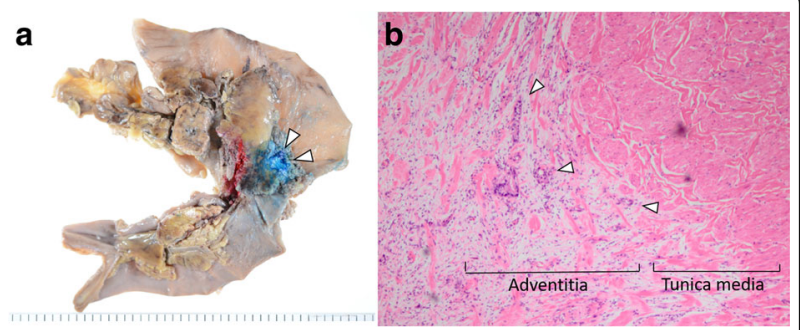

Fig. 3 Macroscopic and microscopic findings of resected specimen of case 1. a Inferior vena cava (IVC) wall was resected together with the pancreatic head (arrowhead). b Tumor invasion of the adventitia of the IVC wall was observed (arrowhead) (hematoxylin and eosin stain, $\times 40$ )
nab-Paclitaxel, $125 \mathrm{mg} / \mathrm{m}^{2}$ ). He was still alive and in stable condition without further tumor progression 12 months after surgery (Fig. 4).

\section{Case 2}

A 59-year-old man with obstructive jaundice was admitted to a different hospital. He was referred to our institution because of possible periampullary malignancies. CT imaging identified an irregular tumor (31 $\mathrm{mm}$ in diameter) in the pancreatic head that extended to the dorsal border of the pancreas (Fig. 5). The tumor was stiffly attached to the IVC wall; therefore, wedge resection of the IVC wall was performed via side clamping of the IVC. Pathological studies of the surgical specimen revealed PDAC directly invading the adventitia of the IVC (Fig. 6). He experienced postoperative pancreatic fistula (International Study Group of Postoperative Pancreatic Fistula grade B) that

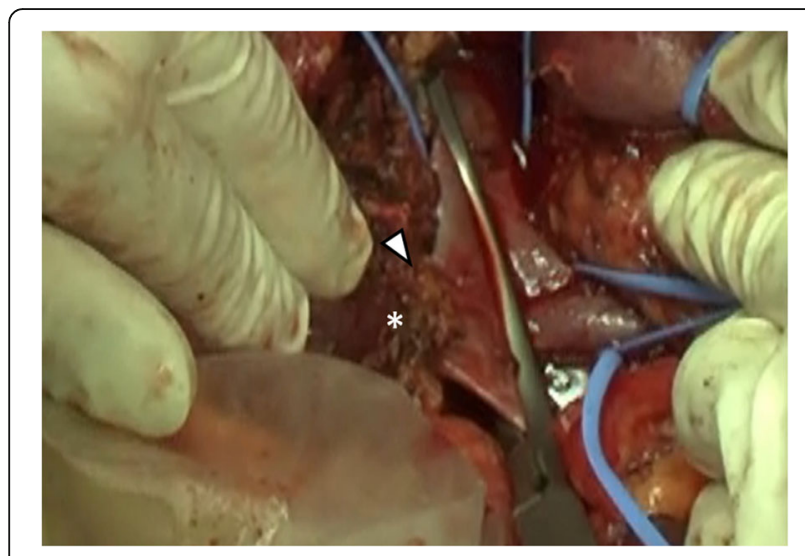

Fig. 2 Intraoperative findings of case 1. Stiff attachment between the tumor (asterisk) and the inferior vena cava (IVC) was identified (arrowhead)

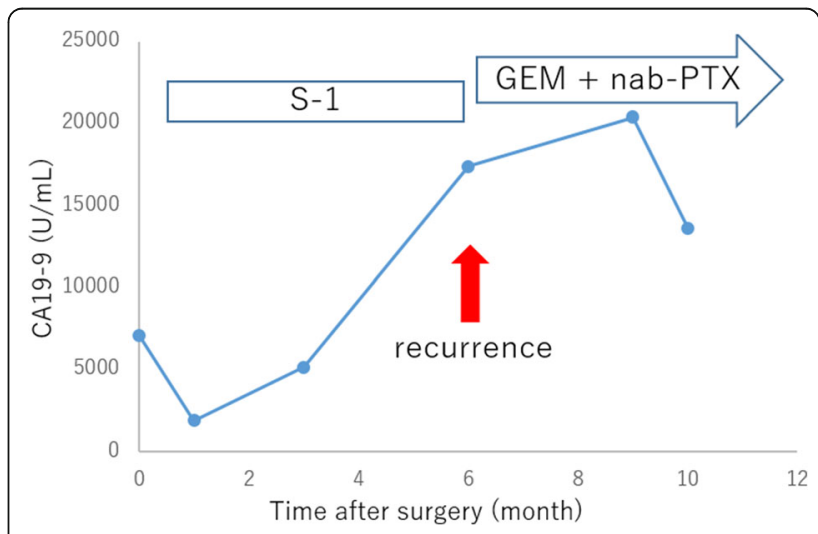

Fig. 4 Changes in serum carbohydrate antigen 19-9 (CA19-9) level associated with postoperative chemotherapy in case 1. CA19-9 level of postoperative month 0 shown in the graph indicates the preoperative CA19-9 level 


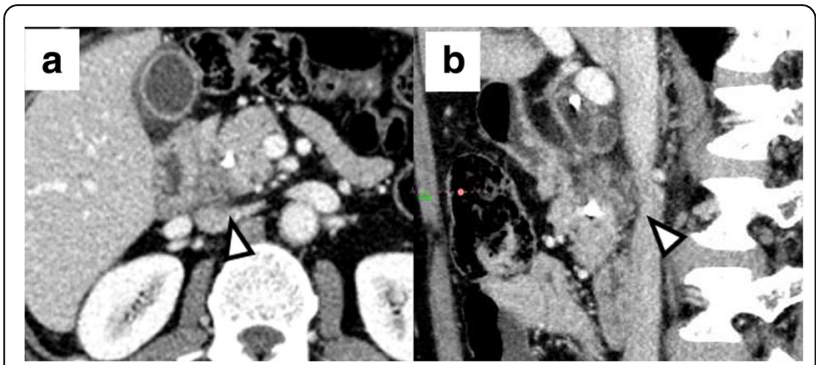

Fig. 5 Preoperative computed tomography $(\mathrm{CT})$ imaging of case 2. a An irregular tumor was identified in the pancreatic head and extended to the dorsal border of the pancreas (arrowhead). $\mathbf{b}$ Sagittal multi-planar reformation showed that the lesion adhered to and deformed the inferior vena cava (IVC) improved with conservative therapy and was discharged on POD 29 [6]. Complications related to IVC resection did not occur during follow-up. He underwent adjuvant systemic chemotherapy ( $-1 ; 120 \mathrm{mg} /$ day) for 6 months and was still alive without tumor recurrence 26 months after surgery (Fig. 7).

\section{Case 3}

A 68-year-old woman with epigastric pain and jaundice presented to a different hospital but was later referred to our institution. CT imaging showed a hypovascular tumor (29 $\mathrm{mm}$ in diameter) in the pancreatic head (Fig. 8). The tumor widely contacted the IVC wall; however, there were no signs of obvious invasion. PDAC was suspected and she underwent PD. Intraoperative findings revealed a stiff attachment between the tumor and the IVC. Wedge resection of the IVC wall was performed using side clamping. In addition, combined resection and reconstruction of the SMV was performed because of suspicion for SMV invasion. Pathological

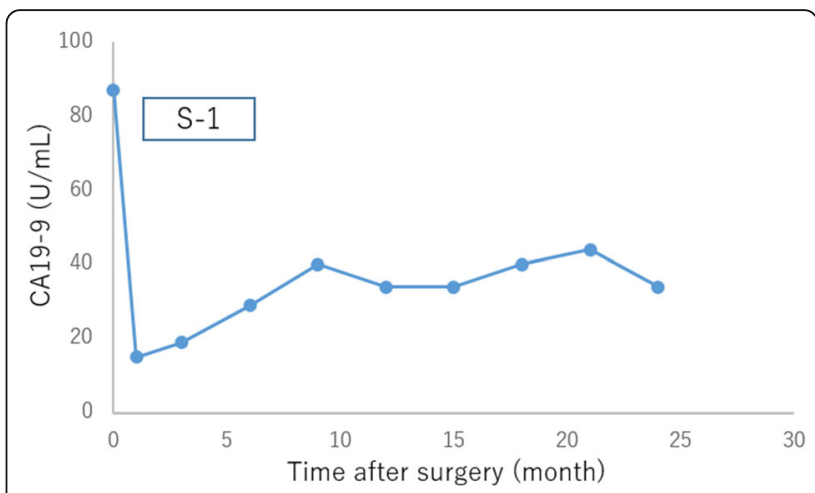

Fig. 7 Changes in serum carbohydrate antigen 19-9 (CA19-9) level associated with postoperative chemotherapy in case 2. CA19-9 level of postoperative month 0 shown in the graph indicates the preoperative CA19-9 level

findings of the surgical specimen revealed tumor infiltration of the adventitia of the IVC (Fig. 9). Surgical margins including IVC resection end were negative; however, tumor metastases to the para-aortic lymph nodes were identified. The postoperative course was uneventful, and she underwent systemic chemotherapy (Gemcitabine, $1000 \mathrm{mg} / \mathrm{m}^{2}$ ) beginning on POD 34. She experienced multiple liver metastases, bone metastasis 10 months after surgery, and died 16 months after recurrence (Fig. 10).

\section{Discussion}

PDAC often infiltrates the adjacent major vasculatures; however, direct invasion of the IVC is rare. We experienced 3 cases of PDAC directly invading the IVC wall among a total of 212 cases of pancreatic resection for PDAC presented during the same period (1.4\%). This

Fig. 6 Macroscopic and microscopic findings of the resected specimen of case 2. a The inferior vena cava (IVC) wall was resected together with the pancreatic head and separated through the pathological examination (arrowhead). Asterisk shows the initial site of the resected IVC wall. $\mathbf{b}$ Tumor invasion of the adventitia of the IVC wall was observed (arrowhead) (hematoxylin and eosin stain, $\times 40$ )
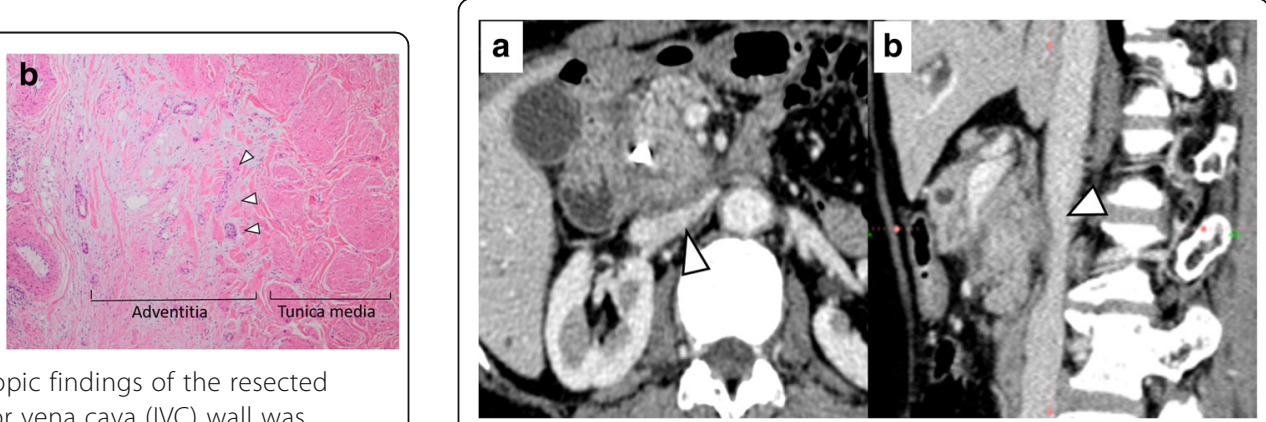

Fig. 8 Preoperative computed tomography (CT) imaging of case 3 . a Dense soft tissue connected to the pancreatic lesion widely contacting the ventral surface of the inferior vena cava (IVC) (arrowhead). b. Sagittal multi-planar reformation showed that the lesion adhered to and deformed the IVC 


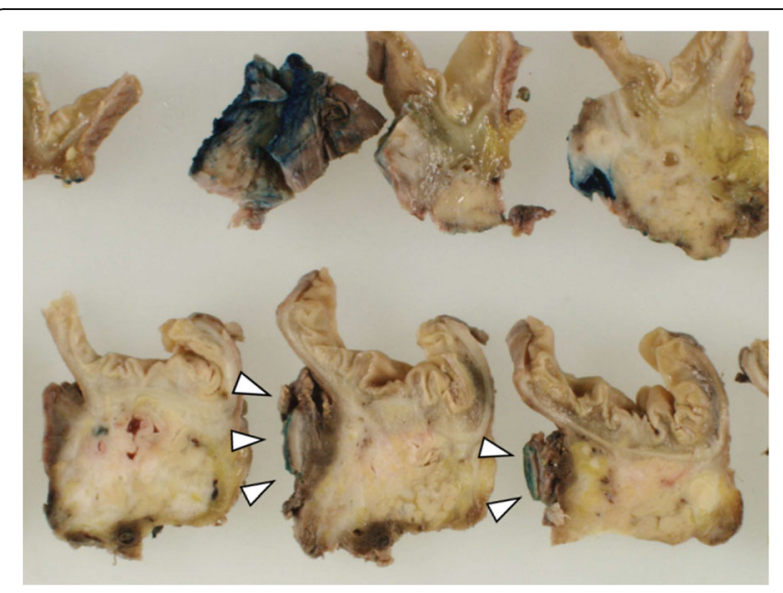

Fig. 9 Macroscopic findings of resected specimen of case 2. The inferior vena cava (IVC) wall was resected together with the pancreatic head (arrowhead)

observation is consistent with a previous study by Kitagawa and colleagues that reported 2 of 140 resected cases of PDAC (1.4\%) with IVC invasion during pathologic studies [7]. Clinical features of these cases are presented in Table 1.

The efficacy of concomitant IVC resection for patients with PDAC is unclear. Among the 3 cases reported in this study, surgical procedures were performed safely and no patient experienced complications related to IVC resection. Regarding oncological aspects, all three patients achieved negative surgical margins. Although one patient was identified to have para-aortic lymph node metastasis on pathological study, postoperative survival

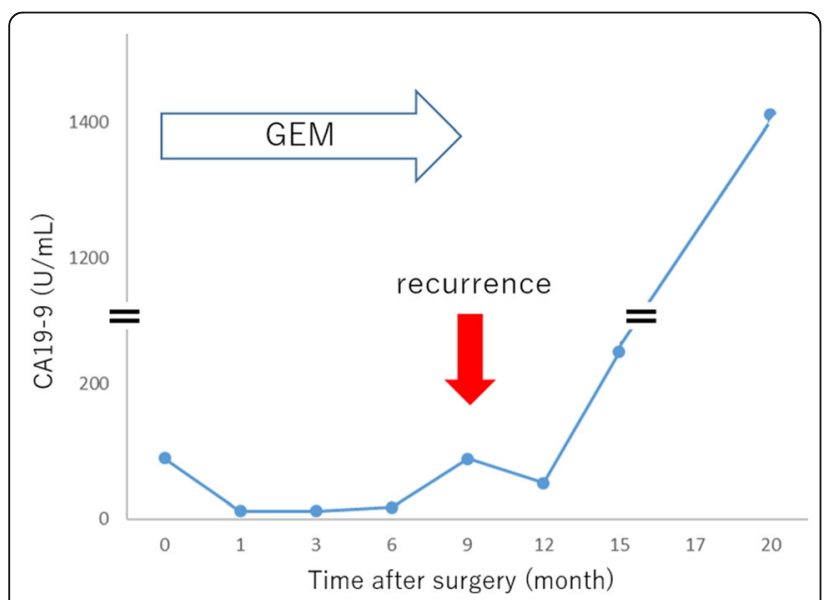

Fig. 10 Changes in serum carbohydrate antigen 19-9 (CA19-9) level associated with postoperative chemotherapy in case 2. CA19-9 level of postoperative month 0 shown in the graph indicates the preoperative CA19-9 level of our cases compared favorably with those of patients with other borderline resectable cases; the reported overall survival time after surgery for those with borderline resectable PDAC is almost 20 months $[8,9]$. Moriura et al. reported two cases of PDAC invading IVC wall during IVC resection for hepato-biliarypancreatic malignancies [10]. The reported prognosis of these two cases was dismal (death due to tumor recurrence 5 and 8 months after surgery, respectively); however, these cases were advanced tumors which needed total pancreatectomy or multivisceral resection and were difficult to simply compare with our cases. Nah et al. also reported a case of PDAC invading IVC wall; however, follow-up period of 8 months was not enough to evaluate postoperative prognosis [11]. Further, more such cases need to be reported in order to elucidate the prognostic impact of IVC invasion.

Kocher's maneuver is usually performed during PD; surgeons dissect between the pancreatic head and IVC. Kitagawa et al. reported that only $3 \%$ of the PDAC directly infiltrates beyond the fusion fascia, which covers the IVC [7]. However, when tumors directly invade the IVC wall, dissecting between the IVC and pancreatic head may cause tumor remnants or seeding due to cutting into the tumor. Because of the desmoplastic nature of PDAC, it is difficult to distinguish fibrous adhesions from tumor involvement intraoperatively. A preoperative predictor of IVC invasion is required; however, there are no criteria that can effectively predict IVC invasion with preoperative imaging studies. According to the NCCN guidelines, PDAC in contact with the IVC is defined as borderline resectable. This definition may lead to a false-positive diagnosis of IVC invasion; PDAC in the pancreatic head occasionally contacts the IVC wall even when IVC invasion is negative. When retrospectively reviewed, the dense soft tissue of the infiltrating tumors widely contacted the IVC wall, and the IVC was deformed on sagittal imaging (Figs. 1b, 3b, and 4b). In cases of tumors contacting the IVC, evaluating the sagittal sections of CT images may help in the diagnosis of IVC invasion preoperatively.

In the case of PDAC largely invading the IVC on preoperative imaging, the indication for surgery should be determined more carefully. In the literature, severe complications such as massive bleeding, gas embolism, venous stenosis, and thrombosis are predominantly associated with segmental resection of the IVC in the setting of liver resection $[12,13]$. Additionally, the oncologic significance of massive IVC invasion is unknown. There can be a potential risk of early pulmonary metastasis in cases of tumors infiltrating the intima of the IVC. 
Table 1 Clinical features of three cases of PDAC invading the IVC

\begin{tabular}{|c|c|c|c|c|c|c|c|c|c|c|c|}
\hline Case & $\begin{array}{l}\text { Age, } \\
\text { years }\end{array}$ & Sex & $\begin{array}{l}\text { Preoperative } \\
\text { CA19-9, U/ml }\end{array}$ & $\begin{array}{l}\text { Operative } \\
\text { procedures }\end{array}$ & $\begin{array}{l}\text { Operation } \\
\text { time }\end{array}$ & Bleeding, $g$ & Tumor size, $\mathrm{cm}$ & $\begin{array}{l}\text { Lymph node } \\
\text { metastasis }\end{array}$ & $\begin{array}{l}\text { Tumor } \\
\text { differentiation }\end{array}$ & $\begin{array}{l}\text { OS, } \\
\text { months }\end{array}$ & $\begin{array}{l}\text { Alive/ } \\
\text { dead }\end{array}$ \\
\hline 1 & 50 & $M$ & 7075 & $P D+I V C R$ & $8 \mathrm{~h}, 26 \mathrm{~min}$ & 225 & 4.8 & Positive & Well to moderate & 12 & $\overline{\text { Alive }}$ \\
\hline 2 & 59 & M & 87 & $P D+I V C R$ & $9 \mathrm{~h}, 45 \mathrm{~min}$ & 690 & 3.1 & Positive & Moderate & 26 & Alive \\
\hline 3 & 68 & $\mathrm{~F}$ & 90 & $P D+I V C R+S M V R$ & $12 \mathrm{~h}, 32 \mathrm{~min}$ & 2300 & 4.5 & Positive & Moderate & 26 & Dead \\
\hline
\end{tabular}

PDAC pancreatic ductal adenocarcinoma, IVC inferior vena cava, $M$ male, $F$ female, $C A 19-9$ carcinoembryonic antigen $19-9, P D$ pancreatoduodenectomy, IVCR inferior vena cava resection, SMVR superior mesenteric vein resection, OS overall survival

\section{Conclusions}

Resected cases of PDAC directly invading the IVC are rare. PD along with wedge resection of the IVC wall for patients with PDAC directly invading the adventitia of the IVC can be performed safely. Further accumulation of cases is needed to elucidate the prognostic impact of IVC invasion.

\section{Abbreviations}

CA19-9: Carbohydrate antigen 19-9; CT: Computed tomography; IVC: Inferior vena cava; PD: Pancreatoduodenectomy; PDAC: Pancreatic ductal

adenocarcinoma; POD: Postoperative day; PV: Portal vein

\section{Acknowledgements}

We thank Editage (www.editage.jp) for the English language editing.

\section{Funding}

The authors declare that this study was not funded externally.

\section{Authors' contributions}

TM wrote the manuscript and performed the literature search. TG and TH reviewed and edited the manuscript. KS supervised TM in assessing CT images. MK, TA, TF, and YK supervised the study. TM, ST, TG, MT, SA, and TH treated and observed the patients. All authors read and approved the final manuscript.

\section{Competing interests}

The authors declare that they have no competing interests.

\section{Consent for publication}

When obtaining informed consent for surgical procedures, general consent for publication and presentation was obtained from the patients.

\section{Publisher's Note}

Springer Nature remains neutral with regard to jurisdictional claims in published maps and institutional affiliations.

Received: 16 January 2017 Accepted: 15 May 2017

Published online: 23 May 2017

\section{References}

1. Cucchetti A, Ercolani G, Taffurelli G, et al. A comprehensive analysis on expected years of life lost due to pancreatic cancer. Pancreatology. 2016;16:449-53.

2. Paulson AS, Tran Cao HS, Tempero MA, Lowy AM. Therapeutic advances in pancreatic cancer. Gastroenterology. 2013;144:1316-26.

3. Bockhorn M, Uzunoglu FG, Adham M, International Study Group of Pancreatic Surgery, et al. Borderline resectable pancreatic cancer: a consensus statement by the International Study Group of Pancreatic Surgery (ISGPS). Surgery. 2014;155:977-88.

4. Barreto SG, Windsor JA. Justifying vein resection with pancreatoduodenectomy. Lancet Oncol. 2016:17:e118-24.

5. National Comprehensive Cancer Network. NCCN clinical practice guidelines in oncology (NCCN Guidelines). Pancreatic adenocarcinoma. Version 1. 2017 http://www.nccn.org/professionals/physician_gls/f_guidelines.asp.
6. Bassi C, Dervenis C, Butturini G, International Study Group on Pancreatic Fistula Definition, et al. Postoperative pancreatic fistula: an international study group (ISGPF) definition. Surgery. 2005;138:8-13.

7. Kitagawa H, Tajima H, Nakagawara $\mathrm{H}$, et al. The retropancreatic fusion fascia acts as a barrier against infiltration by pancreatic carcinoma. Mol Clin Oncol. 2013;1:418-22.

8. Katz MH, Pisters PW, Evans DB, et al. Borderline resectable pancreatic cancer: the importance of this emerging stage of disease. J Am Coll Surg. 2008;206: 833-46.

9. Takahashi S, Kinoshita T, Konishi M, et al. Borderline resectable pancreatic cancer: rationale for multidisciplinary treatment. J Hepatobiliary Pancreat Sci. 2011:18:567-74.

10. Moriura S, Nimura Y, Hayakawa N, et al. Combined resection of the inferior vena cava for hepato-biliary and pancreatic malignancies. Hepatogastroenterology. 1990;37:253-5.

11. Nah YW, Park SJ, Yi JH, Bang SJ. Combined inferior vena cava resection during pancreatoduodenectomy. Korean J Hepatobiliary Pancreat Surg. 2009;13(4):301-6.

12. Hemming AW, Mekeel KL, Zendejas I, Kim RD, Sicklick JK, Reed Al. Resection of the liver and inferior vena cava for hepatic malignancy. J Am Coll Surg. 2013:217:115-24.

13. Nuzzo G, Giordano M, Giuliante F, Lopez-Ben S, Albiol M, Figueras J. Complex liver resection for hepatic tumours involving the inferior vena cava. Eur J Surg Oncol. 2011;37:921-7.

\section{Submit your manuscript to a SpringerOpen ${ }^{\circ}$ journal and benefit from:}

- Convenient online submission

- Rigorous peer review

- Open access: articles freely available online

- High visibility within the field

- Retaining the copyright to your article

Submit your next manuscript at $\boldsymbol{\nabla}$ springeropen.com 